\title{
Three-dimensional finite element mesh generation from measured data of a stamping die
}

\author{
Cheng-En Tsai, Fuh-Kuo Chen* \\ Department of Mechanical Engineering, National Taiwan University, Section 4, No. 1, Roosevelt Road, Taipei 10764, Taiwan, ROC
}

\begin{abstract}
An efficient approach was proposed to generate a three-dimensional (3D) finite element mesh system from the measured data of a stamping die, and a computer software was also developed based on the proposed approach in the present study. The concept of a checker with regular grids was used as a template to sort the tremendous number of measured data into a regular pattern with fewer data. The Bezier and B-spline surfaces were then adopted to smooth the data to implicitly represent the stamping die surface, followed by a special procedure to construct the mesh from the treated data. In the developed software, the desired numbers of nodes and elements, aspect ratio, the maximum allowable curvature, and the allowable error can be specified to obtain an optimal mesh system from the measured data. The validity of the proposed approach and the developed software was demonstrated by three examples. The developed software will be of help to the designers of stamping dies.
\end{abstract}

(c) 2003 Elsevier B.V. All rights reserved.

Keywords: 3D finite element mesh generation; Measured data; Stamping die; Aspect ratio; Bezier and B-spline surfaces

\section{Introduction}

Due to the advance of computer science, the reverse engineering method has been widely used in the die and mold industry. In most cases, the reverse engineering only means to reproduce an existing part using the scanning technology and the CAD/CAM tools to help the manufacturing process. However, a complete reverse engineering may also include the functional analysis of the part to ensure a better design than that of the existing one. The functional analysis is usually performed with the help of a computer-aided engineering (CAE) software. This trend brings the CAE technology to gain its popularity in the die and mold industry recently. Most CAE software is based on the finite element method, and the mesh generation is the fundamental to the analysis.

In the reverse engineering with a stamping die design involved, the surface of the stamping die is measured first by a scanning device, and the measured data are then used to construct the die geometry with a CAD software. The CAD file can be used to build up the finite element mesh system when a CAE analysis is required. However, the process time could be shortened if the finite element mesh is generated directly from the measured data instead of the CAD file. In

\footnotetext{
* Corresponding author. Tel.: +886-2-3621-522; fax: +886-2-3631-755. E-mail address: fkchen@ccms.ntu.edu.tw (F.-K. Chen).
}

the finite element analysis, a sound mesh system leads to a reliable result. Hence, the method of approach to construct a sound finite element mesh system from the measured data is essential to the success of the finite element analysis.

In a three-dimensional (3D) finite element analysis of a stamping process, mesh systems are required for both the sheet blank and the stamping dies. The mesh system of the sheet blank is used to calculate the plastic deformation of the sheet during the stamping process, affecting the accuracy of the results and the computation time. Since the sheet blank is flat and its geometry is usually not complicate, the mesh generation is not difficult and can be completed with any CAD software. As for the mesh system of the stamping die, it is used to describe the geometry of the stamping die and determine the contact condition only, and is not involved with deformation calculation. However, due to the complex geometry of die shape, the mesh generation for the stamping die from the CAD file is usually very time consuming, not to mention from the measured data.

In the research reported, most of them were about two-dimensional mesh system generation [1-3], and more and more about three-dimensional mesh generation were reported in the recent years [4-6]. But most of them were about building up the mesh system of the blank, seldom were the reports about mesh generation of the stamping die. In the present study, an efficient approach of constructing the mesh system of the stamping die from the measured data 
was proposed. In this approach, the measured data were first sorted into a regular pattern, followed by the methods of surface-fitting and surface-smoothing to form an sound grid system. The grid system was then used to build up the mesh system with a selected aspect ratio. An error estimation was also performed to ensure a desired mesh system being constructed. Several examples were demonstrated to validate the method of approaches proposed in the present study.

\section{Sorting of the measured data}

The measured data can be classified into two categories: regular type and irregular type. The regular type means that the measured data are located properly with the same distance in both the $x$ - and $y$-direction on the $x-y$ plane. As to the irregular type, the measured data are unevenly distributed depending on the geometry of the stamping die, fewer points being measured on the flat surface and quite a large number of points being required for the curved geometry. Since the measured data of a stamping die are usually used to generate the tool path for the machining purpose using a CAM program, a tremendous number of points are generally measured. However, an efficient finite element mesh does not require such a large number of nodes and corresponding elements. In order to define a criterion for selecting a proper number of measured data to construct the finite element mesh, a checker system with regular grids, as shown in Fig. 1, was proposed as a template to select suitable measured points. Only one of those points in the same grid is selected and the other ones are deleted for each grid. By doing this, a limited number of measured data are selected and rearranged into a regular pattern. The dimension of the grids can be adjusted according to the density of the mesh required.

For the measured data of regular type, it is easy to perform the sorting procedure as long as the dimension of the grids is larger than the distance of the consecutive points, and no grid will be vacant. But for the irregular type data, some grids may not be occupied because of the uneven distribution
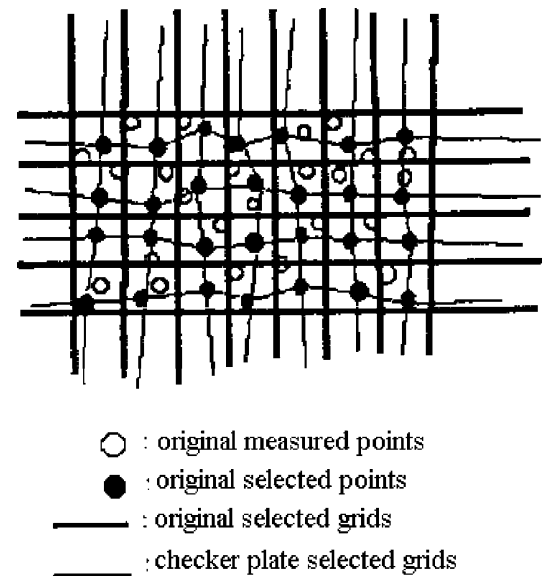

Fig. 1. Grids of checker plate system.

of measured data with pitches larger than the dimension of the grids. In the proposed approach, the vacant grid will be detected and an interpolated point from the neighboring measured data is assigned to that grid.

The measurement of the stamping die boundary is sometimes confusing because that multi-measurement may occur. Since only one of those points in the same grid is selected in the proposed approach, the multi-measured data will not be the problem. But the die boundary may not be exactly represented because the points selected in the grids could not be on the die boundary. One way to remain the original die boundary closely is to use smaller grids at the boundary. In some cases, if the boundary limits are known in advance, then the $x$ - and $y$-coordinate of the points in the grids at the boundary can be assigned to the boundary limits, and the $z$-coordinate can be obtained by interpolation, as shown in Fig. 2.

\section{Surface-fitting and surface-smoothing}

Although the large number of measured data has been sorted in a regular pattern with a reasonable number of points
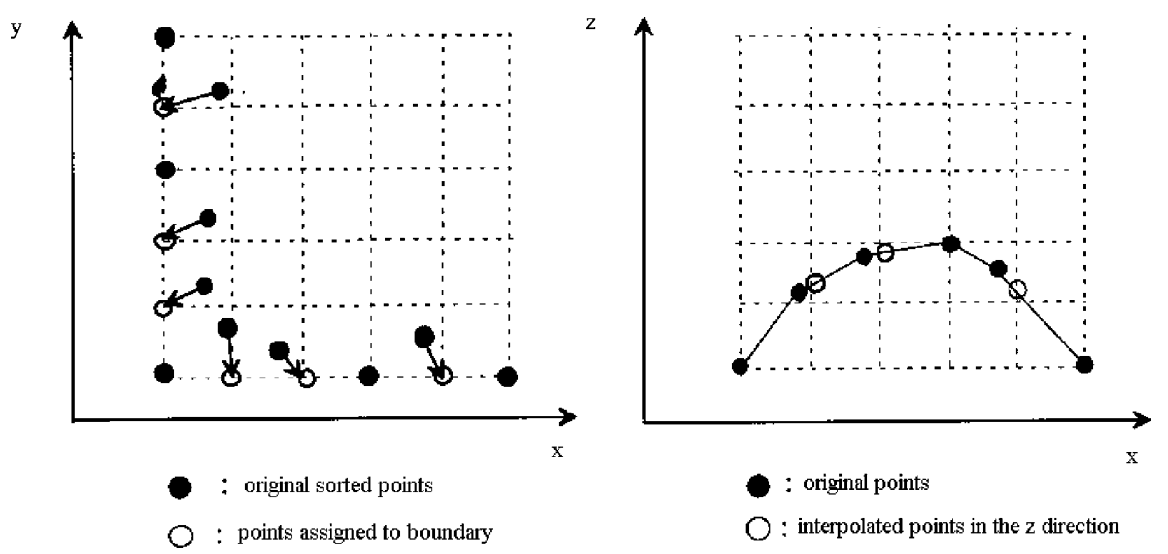

Fig. 2. Treatment of sorted points at boundary. 
using the proposed approach, the sorted data cannot be employed to generate the finite element mesh directly. In order to construct a proper mesh system, the sorted data should represent a smooth die surface implicitly. In the present study, the Bezier surface was adopted to fit sorted data into a surface, and then the fitted surface was smoothened by adding necessary nodes according to the B-spline surface.

The Bezier surface can be represented by the following formulation [7]:

$P(u, w)=\sum_{i=0}^{m} \sum_{j=0}^{n} P_{i j} B_{i, m}(u) B_{j, n}(w), \quad u, w \in[0,1]$

where $u, w$ are parameters, $B_{i, m}(u)=C(m, i) u^{i}(1-u)^{m-i}$, $B_{j, n}(w)=C(n, j) w^{j}(1-w)^{n-j} ; C(m, i)=m ! / i !(m-$ i) !, $C(n, j)=n ! / j !(n-j) !, i, j$ the vertices of the characteristic polyhedron, $m$ the $u$-direction's node number $-1, n$ the $w$-direction's node number $-1, P(u, w)$ the Bezier surface equation, $B_{i, m}(u), B_{j, n}(w)$ the $u, w$-direction's Bernstein Basis function.

The advantages of using the Bezier surface to fit the measured data are as follows:

(a) If the contiguous control points at the boundary of two Bezier surfaces are co-linear on the same line, the first derivatives of the surface functions will be continuous and result in a smooth surface at the boundary of two Bezier surfaces.

(b) Movement of any control point of the Bezier surface will change the whole surface geometry accordingly, and will not loss the smoothness of the original surface, as shown in Fig. 3. However, for the geometry with abrupt change of curvature, the Bezier surface will not remain the same smoothness. To cope with this dilemma, more points will be added to the surface according to the B-spline surface to smooth the surface, as describe below.

(c) In general, a polynomial of degree of three will be sufficient to describe the Bezier surface. This will save the computation time.

As mentioned above, the Bezier surface cannot treat geometry with abrupt change of curvature properly and requires further operation to smoothen the surface. In order

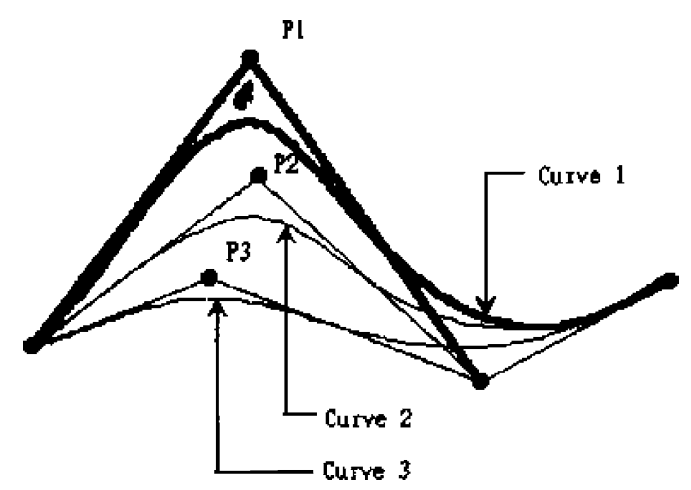

Fig. 3. The Bezier curves. to remain the smoothness of the geometry, more points are required to describe the surface. In the present study, the B-spline surface was adopted to locate the added points by the interpolation from the neighboring data. The abrupt geometry change was first detected by the curvature of the Bezier surface, and was modified by the B-spline surface. The B-spline surface is represented by the following formulation [8]:

$p(u, w)=\sum_{i=0}^{m} \sum_{j=0}^{n} p_{i j} N_{i, k}(u) N_{j, l}(w)$

where $p(u, w)$ is the $\mathrm{B}$-spline surface equation; $u, w$ the two coordinates of the surface; $k, l$ the order of $u, w$-direction $+1 ; p_{i j}$ the vertices of the defining polyhedron; $m$ the control point numbers on the $u$-coordinate $-1 ; n$ the control point numbers on the $w$-coordinate $-1 ; N_{i, k}(u), N_{j, l}(w)$ the blending functions

$$
\begin{aligned}
& N_{i, k}(u)=\frac{u-\alpha_{i}}{\alpha_{i+k-1}-\alpha_{i}} N_{i, k-1}(u)-\frac{u-\alpha_{i+k}}{\alpha_{j+k}-\alpha_{i-1}} N_{i+1, k-1}(u) \\
& N_{i, l}(u)=\left\{\begin{array}{l}
0, i<k \\
i-k+1, k \leq i \leq n \\
n-k+2, i>n
\end{array}\right.
\end{aligned}
$$

$$
\begin{aligned}
N_{i, k}(w)= & \frac{w-\alpha_{j}}{\alpha_{j+l-1}-\alpha_{j}} N_{j, l-1}(w) \\
& -\frac{w-\alpha_{j+1}}{\alpha_{j+1}-\alpha_{j-1}} N_{j+1, l-1}(w)
\end{aligned}
$$

$N_{j, l}(w)=\left\{\begin{array}{l}0, j<l \\ j-l+1,1 \leq j \leq m \\ m-l+2, j>m\end{array}\right.$

The reasons to choose the B-spline surface for adding points are as follows:

(a) Moving any control point of the B-spline surface will only influence neighboring geometry, as shown in Fig. 4. This property is in favor of local modification of a surface.

(b) The non-periodic B-spline surface will be tangential to the line connecting any two neighboring control points.

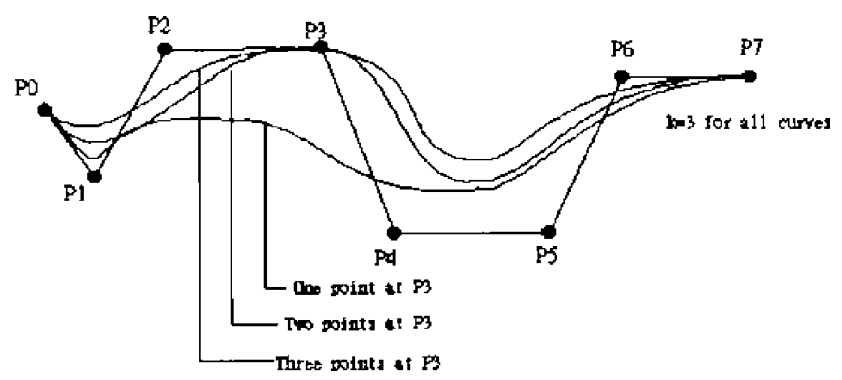

Fig. 4. The B-spline curves. 
So the surface will still pass the control point when more points are added to the neighborhood of that control point.

(c) The order of the B-spline equation is independent of the number of the control points. It helps to economize the computation time.

Although the B-spline surface can describe the abrupt geometry change rather than the Bezier surface, for the surface with a relatively large curvature, the B-spline surface still cannot describe it properly. For such cases, more points are added by the interpolation of the neighboring sorted nodes along the grid sides to remain the smoothness of the geometry. However, the number of points required to be added to the surface depending on the aspect ratio of the mesh defined by the user.

By going through the above procedures, the sorted data can describe the die surface properly and are ready to be used for the mesh generation.

\section{Mesh generation}

Mesh generation plays an important role in the finite element analysis. In the finite element simulation of a stamping process, only the surface of the stamping die, instead of the whole solid die, is required to define the die geometry. Because the mesh system of the stamping die is not involved in the calculation of plastic deformation of the sheet metal, it is not necessary to use high order element to construct the mesh. Hence, the 3-node and 4-node linear shell elements were adopted to generate the mesh system of the stamping die in the present study.

Although the mesh system of a stamping die is not involved in the deformation calculation, the requirements of element angle, aspect ratio, and compatibility are still considered in the present study. In the mesh generation, the nodal coordinates and the nodal connectivity are the fundamentals to the output data.

Since each side of the grid has nodes on it, it is not practical to generate the mesh system just by connecting them directly. Depending on the requirements of the number of element, element angle, and aspect ratio in the mesh, we may need to add new nodes to fulfill the requirements. In the present study, a suitable number of new nodes are added to the grids by interpolation from the original nodes. The numbers of added nodes are in a square pattern, i.e., 1,4 , or 9 , as shown in Fig. 5, depending on the original number of nodes on each side of a grid. The positions of the added nodes will be determined entirely by the original nodes located on each side. To facilitate the procedure of adding nodes, the original grids are projected onto the $x-y$ plane to perform the calculation, and the corresponding $z$-coordinates are interpolated from the coordinates of the related nodes.

The added nodes in the grid are considered as the base nodes, and the mesh is generated by connecting the elements

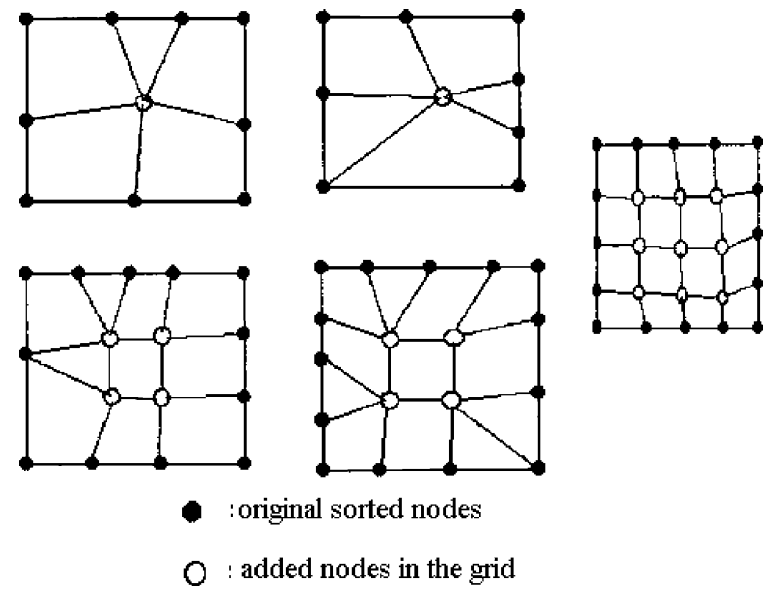

Fig. 5. Three types of adding nodes into grid.

regularly starting from these added nodes. The generated mesh system consists of both triangular and rectangular elements, as shown in Fig. 5. The number of the triangular and the rectangular elements are fully dependent on the nodes in the grid and on each side of the grid.

As connecting the mesh, there are usually two defects may be noticed. One is vacancy and the other is nodal duplication. The vacancy means no element is in that domain which is to be analyzed in the finite element simulation, and the nodal duplication is that a node is repeatedly numbered. In order to avoid these situations, the mesh is generated following the order of the grids. An indicator is also set up to judge whether the element has been designated. If the element has been designated, all nodes of this element will not be designated again to ensure no duplication occurred. Since the mesh is generated following the order of the sorted grids, no vacancy will occur.

\section{Error estimation}

Since the original measured data have been treated by surface-fitting, surface-smoothing, and nodes adding, it is not easy to estimate the error of the generated mesh to the original measured data because they do not have the same base to compare with. In the present study, the difference between the $z$-coordinate of the node of the generated mesh and the original measured point, which have the same $x$ and $y$-coordinate to each other, is defined as the error of that node. The mean error of the whole mesh is given by:

$E=\sqrt{\frac{\sum_{i=1}^{n} e_{i}^{2}}{n}}$

where $E$ is the mean error, $e_{i}$ the error of individual node and $n$ the number of nodes.

In case there is no original measured point corresponding to a certain node in the generated mesh, an interpolation needs to be performed. In order to select the measured points 


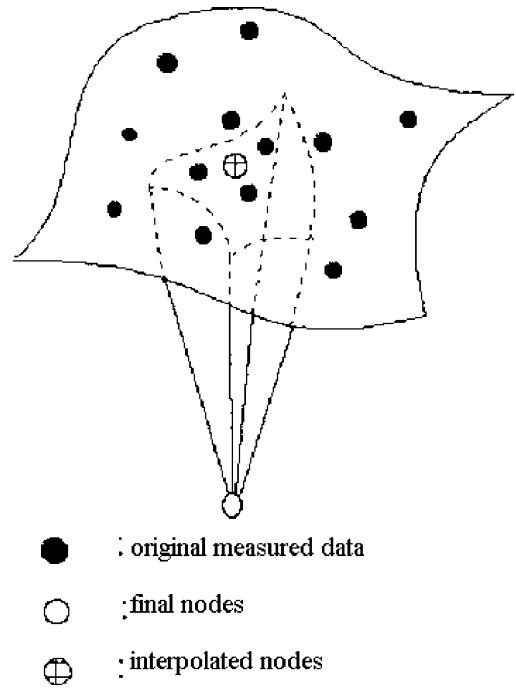

Fig. 6. Selected nodes for the interpolation of the $z$-coordinate.

to be interpolated, an area smaller than the sorted grid is defined. All the measured data enclosed in this area are used to perform the interpolation to determine the $z$-coordinate of the interpolated point that has the same $x$ - and $y$-coordinate as those of the node in the generated mesh, as shown in Fig. 6. If there is no measured data in the defined area, the error is ignored because the position with too few measured data is nearly flat. The reason to choose an area with the side length no larger than the sorted grid sides is that because the corners of the sorted grid have the fairly accurate coordinates. It is easy to interpolate the nodes on each side of the gird using these four nodes. Also, the chosen area larger than the sorted grid side will provoke inaccurate node coordinates sometimes.

The error estimation will help the user to obtain an optimal mesh system by modifying the parameters according to the error allowed.

\section{Examples of application}

A software was developed based on the proposed approach. The developed software can be used to generate a 3D finite element mesh system from the measured data of a stamping die.

The validations of the proposed approach and the developed software are demonstrated by three examples. The first example compares the mesh generated from the measured
Table 1

Conditions of measurement of Example 1

\begin{tabular}{ll}
\hline Number of measured nodes & 2007 \\
Measure pitch in the $x$ - and $y$-direction & $0.5 \mathrm{~mm}$ \\
Measure pitch in the $z$-direction & $0.2 \mathrm{~mm}$ \\
Boundary limit in the $x$-direction & 31.036 to $-11.464 \mathrm{~mm}$ \\
Boundary limit in the $y$-direction & 31.453 to $-11.146 \mathrm{~mm}$
\end{tabular}

Table 2

Parameters and results of Example 1

\begin{tabular}{ll}
\hline Sorting grid dimensions & $2 \mathrm{~mm}$ \\
Aspect ratio & 5 \\
Maximum allowable curvature & $1 / 4 \mathrm{~mm}$ \\
Number of nodes & 1197 \\
Number of elements & 1454 \\
Error $(\%)$ & 1.75 \\
\hline
\end{tabular}

Table 3

Conditions of measurement of Example 2

\begin{tabular}{ll}
\hline Number of measured nodes & 1733 \\
Measure pitch in the $x$-and $y$-direction & $1.0 \mathrm{~mm}$ \\
Measure pitch in the $z$-direction & $0.2 \mathrm{~mm}$ \\
Boundary limit in the $x$-direction & 52.294 to $-1.706 \mathrm{~mm}$ \\
Boundary limit in the $y$-direction & 53.786 to $-2.114 \mathrm{~mm}$
\end{tabular}

Table 4

Parameters and results of Example 2

\begin{tabular}{lll}
\hline & Case 1 & Case 2 \\
\hline Sorting grid dimensions & $2 \mathrm{~mm}$ & $1 \mathrm{~mm}$ \\
Aspect ratio & 5 & 2 \\
Maximum allowable curvature & $1 / 4 \mathrm{~mm}$ & $1 / 4 \mathrm{~mm}$ \\
Number of nodes & 931 & 1685 \\
Number of elements & 1015 & 1834 \\
Error $(\%)$ & 1.34 & 0.85 \\
\hline
\end{tabular}

data by the proposed approach with that constructed by a commercial CAD software, as shown in Fig. 7(a) and (b), respectively. The conditions of the measured data are listed in Table 1, and the parameters chosen for and the results obtained from the proposed approach are given in Table 2. It is seen in both Fig. 7(a) and (b) that the meshes generated by both methods are smooth and compatible. This result confirms the validity of the proposed approach.

The effects of the dimension of the sorted grids and the aspect ratio required in the generated mesh are displayed in Fig. 8(a) and (b), and are demonstrated as the second example. The conditions of the measured data are listed in Table 3, and the parameters set for and the results obtained from the proposed approach are given in Table 4. It is observed

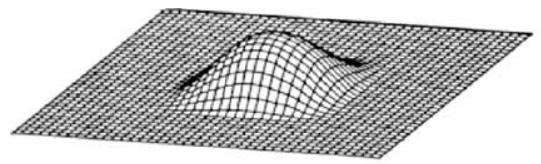

(a) by proposed approach

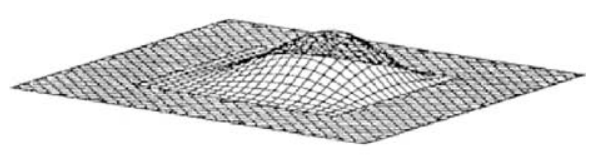

(b) by a $\mathrm{CAD}$ software

Fig. 7. Mesh systems generated in Example 1. 


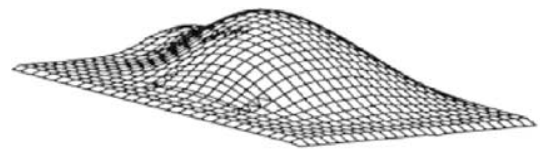

(a) grid size $=2 \mathrm{~mm}$, aspect ratio $=5$

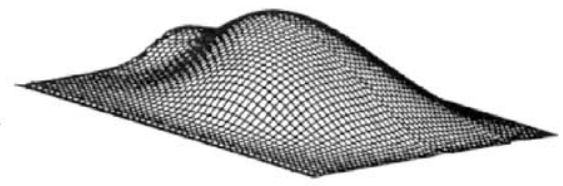

(b) grid size $=1 \mathrm{~mm}$, aspect ratio $=2$

Fig. 8. Effects of grid and aspect ratio on the generated meshes.

Table 5

Conditions of measurement of Example 3

\begin{tabular}{ll}
\hline Number of measured nodes & 4787 \\
Measure pitch in the $x$-and $y$-direction & $3.0 \mathrm{~mm}$ \\
Measure pitch in the $z$-direction & $0.5 \mathrm{~mm}$ \\
Boundary limit in the $x$-direction & 1604 to $-255 \mathrm{~mm}$ \\
Boundary limit in the $y$-direction & 680 to $-10 \mathrm{~mm}$ \\
\hline
\end{tabular}

Table 6

Parameters and results of Example 3

\begin{tabular}{lll}
\hline & Case 1 & Case 2 \\
\hline Sorting grid dimensions & $26 \mathrm{~mm}$ & $26 \mathrm{~mm}$ \\
Aspect ratio & 2 & 2 \\
Maximum allowable curvature & $1 / 5 \mathrm{~mm}$ & $1 / 12 \mathrm{~mm}$ \\
Number of nodes & 2977 & 2884 \\
Number of elements & 4058 & 3796 \\
Error $(\%)$ & 3.02 & 3.04 \\
\hline
\end{tabular}

that the mesh shown in Fig. 8(b), which is constructed with smaller grid and aspect ratio, seems to be smoother than that shown in Fig. 8(a). However, both meshes have about the same order of error, as listed in Table 4. So from the point of view of cost-efficiency, the mesh shown in Fig. 8(a) has fewer nodes and elements but can achieve a reasonable accuracy with less computation time. This example clearly demonstrates the capability of the proposed approach in handling the different aspect ratios required in the generated mesh system.

The third example is the mesh generation of a bathtub. The basic data for the measurement are specified in Table 5. The effect of the maximum allowable curvature in the mesh is examined in this example. The maximum allowable curvatures are chosen to be $1 / 5$ and $1 / 12 \mathrm{~mm}$ for the two cases studied, respectively, and the remaining data are set the same for the both cases. The parameters set for and the results obtained from the proposed approach are given in Table 6 . The meshes generated for the higher allowable curvature and the lower allowable curvature are shown in Fig. 9(a) and (b), respectively. It is noted that the mesh shown in Fig. 9(a) has higher density and seems to be smoother than that shown in Fig. 9(b), which has a smaller allowable curvature. Since the shape of the bathtub does not bear abrupt geometry change, the number of nodes and elements, and the errors of the two meshes differ insignificantly, as shown in Table 6. It implies that the user can specify the maximum allowable curvature to obtain an optimal mesh using the proposed approach.

\section{Concluding remarks}

An efficient approach was proposed in the present study to generate a mesh system from the measured data of a stamping die. The software was then developed based on the proposed approach. With the use of the developed software, an intermediate CAD file is not needed for generating the mesh system from the measured data. In the proposed approach, the number of measured data was first reduced to a reasonable size and sorted into a regular pattern. The sorted data were then treated to implicitly represent smooth surfaces using the Bezier and B-spline surfaces. A number of new nodes were added to smooth the surfaces with abrupt geometry changes by the method of interpolation. A special procedure was also established to construct the mesh system from the treated data. In the software, the desired number of nodes and elements, aspect ratio, the maximum allowable curvature, and the allowable error can be specified to obtain an optimal mesh system.

Three examples of application were demonstrated to validate the proposed approach and the developed software. The compatibility of the mesh generated by the developed software with that generated by a commercial CAD software confirms the validity of the developed software. The capability of the developed software to handle the specifications of the aspect ratio, the maximum allowable curvature, and the allowable error was also demonstrated by the other two

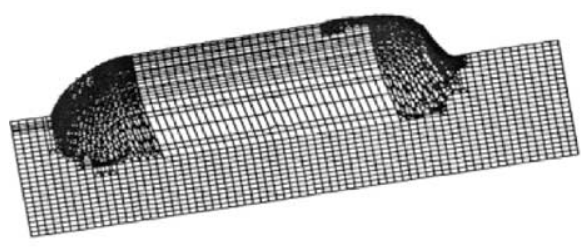

(a) $\max$. allowable curvature $=1 / 5 \mathrm{~mm}$

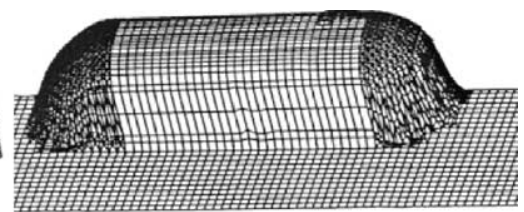

(b) $\max$. allowable curvature $=1 / 12 \mathrm{~mm}$

Fig. 9. Mesh systems generated for a bathtub. 
examples. The developed software will be of help to the designer of stamping dies.

\section{Acknowledgements}

The authors would like to thank the National Science Council of the Republic of China for the financial support under the project \# NSC-87-2213-E-002-008.

\section{References}

[1] M. Witting, S. Burkhardt, Automatic generation of finite difference meshes by an evolutionary algorithm, IEEE Trans. Mag. 32 (3) (1996) $1338-1340$.
[2] S. McFee, D. Giannacopoulos, Optimal discretization based refinement criteria for finite element adaption, IEEE Trans. Mag. 32 (3) (1996) $1357-1360$

[3] K. Ho-Le, Finite element mesh generation methods: a review and classification, Comput. Aided Des. 20 (11) (1988) 27-38.

[4] R.B. Simpson, Automatic local refinement for irregular rectangular meshes, Int. J. Numer. Meth. Eng. 14 (1979) 1665-1678.

[5] S. Motavalli, B. Bldanda, A part image reconstruction system for reverse engineering of design modifications, J. Manuf. Syst. 10 (5) (1991) 383-394.

[6] J.M. Zhou, K.D. Zhou, K.R. Shao, Automatic generation of 3D meshes for complicated solids, IEEE Trans. Mag. 28 (2) (1992) 1759 1762 .

[7] D.L. Taylor, Computer Aided Design, Addison-Wesley, Reading, MA, 1992.

[8] I. Zeid, CAD/CAM Theory and Practice, McGraw-Hill, New York, 1991 